\title{
Self-Reporting Micellar Polymer Nanostructures for Optical Urea Biosensing
}

Sudheesh K. Shukla, Onur Parlak, S.K. Shukla, Sachin Mishra, Anthony Turner and Ashutosh

Tiwari

\section{Linköping University Post Print}

\section{Tweet}

N.B.: When citing this work, cite the original article.

Original Publication:

Sudheesh K. Shukla, Onur Parlak, S.K. Shukla, Sachin Mishra, Anthony Turner and Ashutosh Tiwari, Self-Reporting Micellar Polymer Nanostructures for Optical Urea Biosensing, 2014, Industrial \&amp; Engineering Chemistry Research, (53), 20, 8509-8514.

http://dx.doi.org/10.1021/ie5012799

Copyright: ACS American Chemical Society

http://pubs.acs.org/

Postprint available at: Linköping University Electronic Press

http://urn.kb.se/resolve?urn=urn:nbn:se:liu:diva-107837 


\title{
Self-reporting micellar polymer nanostructures for
}

\section{optical urea biosensing}

Sudheesh K. Shukla ${ }^{1}$, Onur Parlak ${ }^{1}$, Saroj. K. Shukla ${ }^{2}$, Sachin Mishra ${ }^{2}$, Anthony P. F. Turner ${ }^{1}$, Ashutosh Tiwari ${ }^{*}$

${ }^{1}$ Biosensors and Bioelectronics Centre, Department of Physics, Chemistry and Biology, IFMLinköping University, S-58183 Linköping, Sweden

${ }^{2}$ Department of Polymer Science, Bhaskaracharya College of Applied Sciences, University of Delhi, New Delhi 110 075, India

*Corresponding author. Tel.: +4613 2823 95; Fax: +46 13 1375 68; E-mail:

ashutosh.tiwari@liu.se

KEYWORDS. Polymeric nanomicelle, graft co-polymerisation, self-reporting, urea nanobiosensor.

\begin{abstract}
We report the facile fabrication of a self-reporting, highly sensitive and selective optical urea nanobiosensor using chitosan- $g$-polypyrrole (CHIT- $g$-PPy) nanomicelles as a sensing platform. Urease was immobilised on the spherical micellar surface to create an ultra-sensitive self-reporting nanobiosystem for urea. The resulting nanostructures show monodisperse size distribution before and after enzyme loading. The critical micelle concentration of the enzyme-immobilised polymer nanostructure was measured to be $0.49 \mathrm{mg} / \mathrm{L}$ in phosphate buffer at $\mathrm{pH}$ 7.4. The nanobiosensor had a linear optical response to urea concentration ranging
\end{abstract}


from 0.01 to $30 \mathrm{mM}$ with a response time of a few seconds. This promising approach provides a novel methodology for self-reporting bio-assembly over nanostructure polymer micelles and furnishes the basis for fabrication of sensitive and efficient optical nanobiosensors.

\section{INTRODUCTION}

Nanostructured polymer materials have attracted wide attention due to their potential to combine desirable properties of different nanoscale building blocks and improve optical, electronic and mechanical properties. $^{1-3}$ To enhance all these physical and chemical features, several groups have recently produced different classes of polymeric materials with different size and shape, such as polymer nanotubes, nanosheets, nanorods and nanocups ${ }^{4,5}$ However, these structures still suffer from a lack of nanoscale spatial precision, require difficult and time-consuming synthesis processing in bulk and are not always suitable for biological applications. ${ }^{6}$ Moreover, these nanostructures fall short in respect of biological loading such as controlled architecture and operational limitation. In this respect, polymer micellisation is the most powerful and facile approach to achieve high-loading capacity, well-formed micellar structure by using amphiphilic polymers or surfactant to direct biological self-assembly. ${ }^{7}$ These micellar nanostructures have been recently employed in many fields from drug-delivery to biosensors due to their electronic transition, specific surface site and selective reactivity. ${ }^{8-10}$

Chitosan (CHIT) is a well-known and abundantly available natural polymer formed by mollusks,

crustacean and various classes of insects. CHIT has many advantages such as biocompatibility, biodegradability and excellent mechanical strength. ${ }^{11} \mathrm{CHIT}$ is cationic polysaccharide having wide range of potential applications in biosensing, drug delivery, water treatment and pharmaceuticals. CHIT bears both acidic and basic functions and can be easily modified by co-polymerisation, encapsulation and grafting methods. ${ }^{12-14}$ On the other hand, polypyrrole (PPy) is a promising conducting polymer having range of different applications including molecular sensors, gas separation membranes and rechargeable batteries. ${ }^{15}$, 
${ }^{16}$ Although pure PPy has poor solubility, stability and mechanical properties, this can be improved by copolymerisation with biopolymers. ${ }^{17,18}$

Urea is a key analyte in clinical chemistry. Urea, along with creatinine and uric acid, are essential indexes in diagnosing liver and kidney disorders. ${ }^{19-21}$ The normal urea level in blood is 2.5 to $7.5 \mathrm{mM}^{22}$ Measurement of urea is helpful for preventing various diseases such as nephritic syndrome, hepatic failure, urinary track obstruction and renal failure. ${ }^{23}$ Various methods have been reported for the determination of urea in tissue and body fluids including ion chromatography and electrochemical methods, and biosensors are routinely used in hospital laboratories. ${ }^{24,25}$ In this study, we report a facile synthesis and characterisation of chitosan- $g$-polypyrrole (CHIT- $g$-PPy) nanomicelles for the fabrication of a self-reporting optical urea biosensor with a shorter response time and broader dynamic range compared to previous systems. The trustworthy admission of urease onto CHIT-g-PPy nanomicelles is a key element in the proposed design. The CHIT-g-PPy nanomicelles described lay the foundation for a new generation of highly sensitive and reproducible optical urea biosensors for biological samples. Table 1 compares the performance of previously reported enzymatic and non-enzymatic urea sensors. In comparison to other conventional analytical methods, the current biosensing approach has improved detection limits, specificity and response time.

\section{EXPERIMENTAL}

\section{Chemicals}

Chitosan (CHIT, $>75 \%$ deacetylated, $M_{\mathrm{w}}$ 120-150k), pyrrole (99.5\%), ammonium persulphate (APS) (99.5\%) urease (Urs, EC 3.5.1.5, type III from Jack Beans, 29,500 units/g), 1-ethyl-3-(3dimethylaminopropyl) carbodiimide hydrochloride (EDC, 99\%), $N$-hydroxysuccinimide (NHS, 99\%), urea, uric acid, lactic acid, ascorbic acid were purchased from Sigma-Aldrich (St. Louis, MO, USA) and used without further purification. All the supplementary chemicals were of analytical grades and solutions were prepared with Milli-Q water (18.2 M $\Omega$ ). 


\section{Preparation of CHIT and PPy solution}

CHIT solution was prepared by mixing of CHIT flakes (1.0 g) in acetic acid-water mixture (25\% v/v, 100 $\mathrm{mL}, 1 \%, \mathrm{pH} \sim 4)$. The CHIT solution was stirred magnetically for 2 hours at room temperature, yielding a flake-free clear solution of CHIT. Pyrrole solution $(10.0 \mathrm{~mL})$ was prepared by mixing pyrrole in aqueous ammonium persulphate $(0.5 \mathrm{M}, 5.0 \mathrm{~mL})$ at room temperature with constant magnetic stirring for 5 min.

\section{Grafting of PPy onto CHIT}

In a typical run, $10 \mathrm{~mL}$ of flakes free CHIT solution and $1.5 \mathrm{~mL}$ of freshly prepared pyrrole with ammonium persulphate solution were mixed in a $25 \mathrm{~mL}$ vessel. The vessel was sealed under $\mathrm{N}_{2}$ gas and evacuated at room temperature for $24 \mathrm{~h}$. The obtained product was dialysed against deionised water to remove salts and water soluble contaminants, followed by vacuum-drying at $60{ }^{\circ} \mathrm{C}$. The dried copolymer was further extracted successively with m-cresol and dimethyl sulfoxide in a Soxhlet apparatus for $36 \mathrm{~h}$, in order to remove the homopolymer of PPy. The grafting percent $(\% \mathrm{G})$ of PPy onto CHIT was calculated using the following equation: ${ }^{26}$

$$
G \%=\frac{W_{1}-W_{0}}{W_{0}} \times 100
$$

Where, $W_{1}$ is weight of CHIT- $g$-PPy and $W_{0}$ is weight of CHIT.

\section{Preparation of Urs functionalised CHIT-g-PPy micelles}

The Urs functionalised CHIT-g-PPy micelles were prepared by a two-step process. In the first step, CHIT$g$-PPy micelles were produced using membrane dialysis technique and in the second step functionalisation of Urs on CHIT-g-PPy micelles via coupling chemistry was carried out. Typically, the CHIT-g-PPy (40 mg) was dissolved in $3 \mathrm{~mL}$ acid water of $\mathrm{pH} 4.0$ under sonication. Milli-Q water (12 mL) was added dropwise to the resulting mixture. Thereafter, enzyme was covalently immobilised on the CHIT- $g$-PPy micellar structure using EDC/NHS coupling chemistry. Likewise, $50 \mu \mathrm{L}$ Urs was dissolved in MES buffer at pH 5.0 followed by addition of $12 \mathrm{mM}$ EDC and $5 \mathrm{mM}$ NHS with continuous stirring for $30 \mathrm{~min}$ at room 
temperature. Then the solution mixture was added drop wise to the CHIT- $g$-PPy micellar dispersion and left overnight. The resulting Urs/CHIT-g-PPy micellar assembly was dialysed for $48 \mathrm{~h}$ against Milli-Q water using dialysis tubing with a molecular weight cut-off of 2000 Dalton.

\section{Spectrophotometric study}

The Urs/CHIT-PPy nanomicelles were mixed in a $3 \mathrm{~mL}$ phosphate buffer solution (50 mM, pH 7.4) containing $15 \mu \mathrm{L}$ Nessler's reagent and urea solution with varying concentrations. After 3 min of incubation, the absorbance of the colored product, $\mathrm{NH}_{2} \mathrm{Hg}_{2} \mathrm{I}_{3}$, a complex formed as a result of reaction between Nessler's reagent and ammonia, which is produced after the enzymatic hydrolysis of urea at $\lambda_{\max }$ $385 \mathrm{~nm}$, was measured to determine the kinetics of the urease.

\section{Instrumentation}

The photometric studies were carried out using a UV-2501 PC, Shimadzu Corporation Japan, UV-visible spectrophotometer. Fourier transform infrared spectroscopy (FTIR) was performed using a Perkin Elmer RK-1310 spectrophotometer (PerkinElmer, Waltham, MA, USA). The critical micelle concentration (CMC) measurement of the micellar nanostructures was recorded on a fluorescence spectrometer (Photon Technology International Inc., Birmingham, NJ, USA) using pyrene as an indicator. Transmission electron microscopy (TEM) images were obtained using an interface high resolution transmission electron microscopy (HITACHI H-7650, Tokyo, Japan). A TEM sample was prepared by depositing $6 \mu \mathrm{L}$ solution of the nanomicelles (ultrasonically dispersed in THF) on a copper grid. The size of Urs/CHIT-g-PPy micellar nanostructures was determined by dynamic light scattering (DLS) using a Beckman Coulter PCS submicron particle size analyser at $90^{\circ}$. The concentration of the CHIT- $g$-PPy and Urs/CHIT- $g$-PPy solution was $0.35 \mathrm{mg} / \mathrm{mL}$. The \% total wet- weight was calculated using following equation:

$$
S=\frac{W_{w}-W_{d}}{W_{w}} \times 100
$$

where, $W_{\mathrm{w}}$ and $W_{\mathrm{d}}$ is the wet and dry weight of Urs/CHIT- $g$-PPy micelles, respectively. 


\section{Urea nanobiosensor study}

The urea biosensing study was performed using a UV-visible spectrophotometer. Typically, Urs/CHIT- $g$ PPy nanomicelles were prepared in a $3 \mathrm{~mL}$ phosphate buffer solution (50 mM, pH 7.4) and a fixed amount of urea was added to the solution, ranging from 0.01 to $30 \mathrm{mM}$. After $3 \mathrm{~min}$ of incubation, Urs/CHIT- $g$ PPy nanomicelles started to produce ammonia by the enzymatic hydrolysis of urea. The absorbance of the micelle was quantitatively determined at $\lambda_{\max } 385 \mathrm{~nm}$ with respect to urea concentration for each mL of solution. The plot of urea concentration versus absorbance served as a calibration curve for the determination of unknown concentrations of urea in the test samples.

\section{RESULTS AND DISCUSSION}

Scheme 1 represents the overall process for the preparation of enzyme-immobilised polymer micelles for optical urea sensing. The synthesis strategy was to obtain CHIT-g-PPy spherical nanomicelles via covelent bonding and then to conjugate the enzyme on the spherical micelles. This allowed optical sensing of various concentration of urea.

Synthesis and characterisation of CHIT-g-PPy and Urs/CHIT-g-PPy

The CHIT is an amphiphilic polymer, thus the dispersion of pyrrole started the nucleation in polymeric network in the presence APS and resulted in cationic PPy macro radicals. When the concentration of PPy radicals reaches saturation level, their reactivities reduce. After mixing of cationic PPy macro radicals with protonated CHIT solution, they initiate formation of the CHIT macro radicals by the attracting of hydrogen ion from the hydroxyl groups. Finally, graft copolymerisation of PPy macro radicals to CHIT backbone was performed by the charge stabilisation, i.e., $-\mathrm{NH}_{3}{ }^{+}$groups of CHIT protected the amine functions to further covalently attach the urease on the periphery. A black colour CHIT-g-PPy was resulted with 198 G\%. 
The critical micelle concentration (CMC) of CHIT- $g$-PPy micelles was determined by using pyrene as a hydrophobic probe. Fluorescence spectra of the pyrene containing CHIT- $g$-PPy solutions were recorded from 280 to $360 \mathrm{~nm}$ with an emission wavelength of $390 \mathrm{~nm}$ at room temperature. The CMC of the CHIT-g-PPy was measured to be $0.49 \mathrm{mg} / \mathrm{L}$ in PBS at $\mathrm{pH}$ 7.4. The measured CMC value of micelles favours the stability of a micellar structure in an aqueous environment. The size of the CHIT- $g$-PPy and Urs/CHIT-g-PPy micelles was determined by DLS and the results are shown in Figure 1. The micelles exhibited a homogeneous size distribution with a diameter ranging from 80-110 nm (CHIT-g-PPy) and 130160 nm (Urs/CHIT- $g$-PPy). The average hydrodynamic diameters of CHIT-g-PPy and Urs/CHIT- $g$-PPy micelles were measured to be 90 and $145 \mathrm{~nm}$, respectively with a polydispersity index of 0.04 and 0.06 . The increase in average size of Urs/CHIT-g-PPy micelles indicates immobilisation of Urs enzyme on the spherical micelle surface.

The size and morphology of CHIT-g-PPy and Urs/CHIT-g-PPy micelles were further characterised by transmission electron microscope (TEM) (Figure 2). Panel “a” in Figure 2 shows CHIT-g-PPy nanomicelles and illustrates the formation of a multi-component micelle-type structure, due to the successful graft co-polymerisation between CHIT and PPy. It can be seen from the figure that CHIT polymer (shell in gray color) may be covered on the PPy surface. The average size was calculated to be 70 nm, which is very close to the hydrodynamic size measured by DLS (75 nm). Panel "b” in Figure 2 shows a TEM image of a polymer micelle after enzyme immobilisation, showing that the average size of particle significantly increases. In addition, surface modification of the particle with enzyme shows that the synthesised CHIT-g-PPy nanomicelles had homogeneous surface morphology.

The FTIR spectra of the CHIT- $g$-PPy and Urs/CHIT- $g$-PPy are given in Figure 3. The FTIR spectrum of CHIT-g-PPy shows typical absorption bands at $3418 \mathrm{~cm}^{-1}$, which indicates $\mathrm{N}-\mathrm{H}$ and $\mathrm{O}-\mathrm{H}$ stretching, and $2934 \mathrm{~cm}^{-1}$ shows aromatic C-H stretching vibration. In addition, the bands at $2844 \mathrm{~cm}^{-1}$ denotes $\mathrm{C}-\mathrm{H}$ stretching of aliphatic $-\mathrm{CH}_{2}$ groups, and at 1620 and $1486 \mathrm{~cm}^{-1}, \mathrm{C}=\mathrm{O}$ stretching of amide I and II groups, respectively. The vibration band at $1023 \mathrm{~cm}^{-1}$ indicates $\mathrm{C}=\mathrm{O}$ stretching due to the carbonyl 
groups and at 798 and 612, $\mathrm{cm}^{-1}$ show bending vibrations of C-H groups of the grafted PPy onto CHIT backbone. The $\mathrm{O}-\mathrm{H}$ stretching is observed in the range of 3273 to $3600 \mathrm{~cm}^{-1}$. The broadening in this peak depends on the existing groups in the CHIT-g-PPy. The broad and shoulder like peak at $3415 \mathrm{~cm}^{-1}$ is due to the surface hydroxyl groups. Hence, FTIR spectra confirm the grafting of PPy on to CHIT, i.e., formation of CHIT- $g$-PPy. Moreover, the FTIR spectrum of the Urs/ CHIT- $g$-PPy revealed absorption broadening at 3000 to $3600 \mathrm{~cm}^{-1}$. However, the peak broadening at 1600 and $1410 \mathrm{~cm}^{-1}$ corresponds to increasing amide bands due to the covalent attachment of Urs enzymes on the free $-\mathrm{NH}_{2}$ groups of CHIT- $g$-PPy nanomicelles. ${ }^{27}$ Thus the FTIR study shows strong evidence for the immobilisation of Urs on the CHIT- $g$ PPy nanomicelles.

\section{Self-reporting urea biosensing}

The swelling behavior of materials allows the quick diffusion of analyte and provides a surface reaction zone for sensing purposes. The high swelling value of Urs/CHIT-g-PPy nanomicelle (46\%) favours sensingr. It is well known that the diffusion of urea solution is much better in the swollen state. This diffusion generates an instantaneous osmotic pressure gradient across the interface between the sensing platform such as Urs/CHIT-g-PPy nanomicelle and urea solution. This osmotic pressure gradient reversibly affects the equal distribution of ions with concomitant increase in urease efficiency. A photometric response study was carried out to determine the apparent urease activity, stability, effect of interferents and shelf life of Urs/CHIT-g-PPy nanomicelles. The photometric response of this sensor was followed using the wellknown coloured complex formation between Nessler's reagent, $\mathrm{K}_{2} \mathrm{HgI}_{4}$ and ammonia produced by the enzymatic hydrolysis of urea by urease enzyme. ${ }^{28}$ The synthesised Urs/CHIT-g-PPy nanomicelles were immersed in a reaction mixture (in UV-vis cubit) containing $3 \mathrm{~mL}$ of PBS solution ( $\mathrm{pH} \mathrm{7.5),} 15 \mu \mathrm{l}$ of Nessler's reagent solution and $100 \mu \mathrm{L}$ of analyte in varying concentrations (0.01 to $30 \mathrm{mM})$ for $3 \mathrm{~min}$. The urease catalyses the hydrolysis of urea to ammonia which in turns to react with the Nessler's solution $\left(\mathrm{K}_{2} \mathrm{HgI}_{4}\right)$ in order to form a coloured complex $\left(\mathrm{NH}_{2} \mathrm{Hg}_{2} \mathrm{I}_{3}\right)$. By following the absorbance at $385 \mathrm{~nm}$, urea can be quantified and the analytical performance of the sensor determined. The differences between the 
initial and final absorbance value at $385 \mathrm{~nm}$ were plotted as a function of urea concentration, as shown in

Figure 4.

In this pH-responsive Urs/CHIT-g-PPy nanomicelle, CHIT may deacetylate in a basic environment thus enhancing the diffusion rate of ammonia. This is responsible for the doping of PPy grafted chains. Figure 4 shows the change in $\lambda_{\max }$ value with increase in urea concentration. It was observed that the photometric response increases linearly ranging from 0.01 to $30 \mathrm{mM}$. It may be due to the production of ammonia increases the urea concentration and doping level in the Urs/CHIT-g-PPy nanomicelle. The linear regression for urea response of the Urs/CHIT-g-PPy nanomicelles was established with correlation coefficient $\left(R^{2}\right)$ of 0.995 according to the following equation:

$$
\lambda_{\max }=1.11 \times 10^{3}+4.949 \times 10^{-5}[\text { Urea, } \mathrm{mM}]
$$

\section{Effect of interferents}

The effect of interferents i.e. glucose (5 mM), uric acid (0.15 mM), lactic acid (5.5 mM), ascorbic acid (0.1 $\mathrm{mM}$ ) and cholesterol (5.2 mM), respectively, on the Urs/CHIT-PPy nanomicelles response to urea was determined. Negligible effects were observed will all the interferents except uric acid and ascorbic acid, due to the production of free radicals by spontaneous oxidation with the urease enzyme in solution generating superoxide anion radical.

\section{Nanobiosensor performance}

The responsive chemical nature of CHIT is well known and important for biosensing purposes. ${ }^{29,30}$ Usually, biomolecules contain hydroxyl groups which produce hydronium ion (bicarbonate and ammonium ions) after hydrolysis. These generated hydronium ions can be triggered in the presence of ionisable functional groups such as $-\mathrm{COOH},-\mathrm{NH}_{2}$ and become ionised, acquiring charge at a specific $\mathrm{pH}$ value. The polymeric chain has a number of similar functional charged groups, which cause repulsion. Due to this repulsion, polymeric materials expand and change their dimension to reduce the steric hindrance. On the other hand, with change of $\mathrm{pH}$, functional groups reduce their charge and their dimension. Expansion facilitates 
diffusion, which may generate an enhanced and effective impulse in the form of electronic transitions due to the partial ionisation of the polymeric chain. The electronic transitions effectively serve as a tool for the optical monitoring to sense the biomolecules, in this case urea.

A static response time around 12 seconds, with a recovery time of 38 seconds was observed during the sensing experiment. The enhanced sensitivity may be due to availability of urease enzymes at the periphery of Urs/CHIT-g-PPy nanomicelle. It can also be attributed to the high urease functionality onto the CHIT$g$-PPy nanomicelle, especially due to the free $-\mathrm{NH}_{2}$ groups in the CHIT backbone. The storage stability of the Urs/CHIT-g-PPy nanomicelle was measured and similar sensing response was found after it was stored for one-month at $4{ }^{\circ} \mathrm{C}$. Thus Urs/CHIT-g-PPy nanomicelles exhibited a long operational and storage stability. This new type of urease functionalised CHIT-g-PPy nanomicelle demonstrated a shorter response time with broader detection range in comparison to those reported previously, as shown in Table 1.

\section{CONCLUSION}

A self-reporting optical urea nanobiosensor based on Urs/CHIT-g-PPy nanomicelles was successfully fabricated and evaluated. Urease was immobilised onto amphiphilic PPy grafted CHIT, which served as a nano-matrix for the development of a urea biosensor. The copolymerised CHIT-g-PPy hybrid nanomicellar structure offered a high level of enzyme immobilization leading to highly stable Urs/CHIT-g-PPy nanomicelles. This optical urea biosensor showed a linear response to urea concentrations ranging from 0.01 to $30 \mathrm{mM}$ and exhibited a sensitivity of $0.25 \mu \mathrm{M}$ with a response time of $12 \mathrm{~s}$. In this study, CHIT was grafted with PPy and fabricated as a pH-responsive nanomicelle to improve the osmotic gradient for analyte without the loss of optical performance. These results indicate that the proposed miceller nanosystem could be a promising option for the development of a range of self-reporting oxidase-based biosensors in the future. Owing to the selective hydrolysis of the urea, the proposed nanoreactor could be actively take part in the development of artificial kidney. 


\section{ACKNOWLEDGEMENT}

Authors wish to thank the European Commission (PIIF-GA-2009-254955) and UGC, India (MRP-83(47)/2011) for generous support to carry out this research.

SUPPORTING INFORMATION

This information is available free of charge via the Internet at http://pubs.acs.org/.

\section{REFERENCE}

(1) Whitesides, G. M.; Mathias, J. P.; Seto, C. T. Molecular self-assembly and nanochemistry - A chemical strategy for the synthesis of nanostructures. Science 1991, 254, 1312-1319.

(2) Stupp, S. I.; LeBonheur, V.; Walker, K.; Li, L. S.; Huggins, K. E.; Keser, M.; Amstutz, A. Supramolecular materials: Self-organized nanostructures. Science 1997, 276, 384-389.

(3) O'Reilly, R. K.; Hawker, C. J.; Wooley, K. L. Cross-linked block copolymer micelles: functional nanostructures of great potential and versatility. Chem. Soc. Rev. 2006, 35, 1068-1083.

(4) Elsabahy, M.; Wooley, K. L. Design of polymeric nanoparticles for biomedical delivery applications. Chem. Soc. Rev. 2012, 41, 2545-2561.

(5) Owen, S. C.; Chan, D. P. Y.; Shoichet, M. S. Polymeric micelle stability. Nano Today 2012, 7, 53-65.

(6). Turner, A. P. F. Biosensors: sense and sensibility. Chem. Soc. Rev. 2013, 42, 3184-3196.

(7) Lukyanov, A. N.; Torchilin, V. P. Micelles from lipid derivatives of water-soluble polymers as delivery systems for poorly soluble drugs. Adv. Drug Delivery Rev. 2004, 56, 12731289.

(8) Simnick, A. J.; Lim, D. W.; Chow, D.; Chilkoti, A. Biomedical and biotechnological applications of elastin-like polypeptides. Polym. Rev. 2007, 47, 121-154.

(9) Tong, W.; Song, X.; Gao, C. Layer-by-layer assembly of microcapsules and their biomedical applications. Chem. Soc. Rev. 2012, 41, 6103-6124.

(10) Hu, J.; Liu, S. Responsive Polymers for Detection and Sensing Applications: Current Status and Future Developments. Macromolecules 2010, 43, 8315-8330.

(11) Kumar, M.; Muzzarelli, R. A. A.; Muzzarelli, C.; Sashiwa, H.; Domb, A. J. Chitosan chemistry and pharmaceutical perspectives. Chem. Rev. 2004, 104, 6017-6084.

(12) Parlak, O.; Tiwari, A.; Turner, A. P. F.; Tiwari, A. Template-directed hierarchical selfassembly of graphene based hybrid structure for electrochemical biosensing. Biosens. Bioelectron. 2013, 49, 53-62.

(13) Krajewska, B. Application of chitin- and chitosan-based materials for enzyme immobilizations: a review. Enzyme Microb. Tech. 2004, 35, 126-139. 
(14) Horzum, N.; Demir, M. M.; Nairat, M.; Shahwan, T. Chitosan fiber-supported zero-valent iron nanoparticles as a novel sorbent for sequestration of inorganic arsenic. RSC Adv. 2013, 3, 7828-7837.

(15) Ramanavicius, A.; Kausaite, A.; Ramanaviciene, A. Biofuel cell based on direct bioelectrocatalysis. Biosens. Bioelectron 2005, 20, 1962-1967.

(16) KorriYoussoufi, H.; Garnier, F.; Srivastava, P.; Godillot, P.; Yassar, A. Toward bioelectronics: Specific DNA recognition based on an oligonucleotide-functionalized polypyrrole. Journal of the American Chemical Society 1997, 119, 7388-7389.

(17) Aranda, P.; Darder, M.; Fernandez-Saavedra, R.; Lopez-Blanco, M.; Ruiz-Hitzky, E. Relevance of polymer- and biopolymer-clay nanocomposites in electrochemical and electroanalytical applications. Thin Sol. Films 2006, 495, 104-112.

(18) Lakard, B.; Magnin, D.; Deschaume, O.; Vanlancker, G.; Glinel, M.; DemoustierChampagne, S.; Nysten, B.; Jonas, A. M.; Bertrand, P.; Yunus, S. Urea potentiometric enzymatic biosensor based on charged biopolymers and electrodeposited polyaniline. Biosens Bioelectron 2011, 26, 4139-4145.

(19) Sangodkar, H.; Sukeerthi, S.; Srinivasa, R. S.; Lal, R.; Contractor, A. Q. A biosensor array based on polyaniline. Anal. Chem. 1996, 68, 779-783.

(20) Sharma, A. C.; Jana, T.; Kesavamoorthy, R.; Shi, L. J.; Virji, M. A.; Finegold, D. N.; Asher, S. A. A general photonic crystal sensing motif: Creatinine in bodily fluids. J. Am. Chem. Soc. 2004, 126, 2971-2977.

(21) Nikoleli, G. P.; Nikolelis, D. P.; Methenitis, C. Construction of a simple optical sensor based on air stable lipid film with incorporated urease for the rapid detection of urea in milk. Anal. Chim. Act. 2010, 675, 58-63.

(22) Abel, A. P.; Weller, M. G.; Duveneck, G. L.; Ehrat, M.; Widmer, H. M. Fiber-optic evanescent wave biosensor for the detection of oligonucleotides. Anal. Chem. 1996, 68, 29052912.

(23) Evtugyn, G. A.; Budnikov, H. C.; Nikolskaya, E. B. Sensitivity and selectivity of electrochemical enzyme sensors for inhibitor determination. Talanta 1998, 46, 465-484.

(24) Zhang, Y. Y.; Tadigadapa, S. Calorimetric biosensors with integrated microfluidic channels. Biosens Bioelectron 2004, 19, 1733-1743.

(25) Nikoleli, G. P.; Israr, M. Q.; Tzamtzis, N.; Nikolelis, D. P.; Willander, M.; Psaroudakis, N. Structural Characterization of Graphene Nanosheets for Miniaturization of Potentiometric Urea Lipid Film Based Biosensors. Electroanal. 2012, 24, 1285-1295.

(26) Shukla, S. K.; Deshpande, S. R.; Shukla, S. K.; Tiwari, A. Fabrication of a tunable glucose biosensor based on zinc oxide/chitosan-graft-poly(vinyl alcohol) core-shell nanocomposite. Talanta 2012, 99, 283-287.

(27) Tiwari, A.; Singh, V. Synthesis and characterization of electrical conducting chitosangraft-polyaniline. Express Poly. Let. 2007, 1, 308-317.

(28) Li, H.; Luo, R. M. Modeling and characterization of glucose-sensitive hydrogel: Effect of Young's modulus. Biosens Bioelectron 2009, 24, 3630-3636.

(29) Ding, L.; Hao, C.; Xue, Y. D.; Ju, H. X. A bio-inspired support of gold nanoparticleschitosan nanocomposites gel for immobilization and electrochemical study of K562 leukemia cells. Biomacromol. 2007, 8, 1341-1346.

(30) Parlak, O.; Turner, A. P. F.; Tiwari, A. On/Off-Switchable Zipper-Like Bioelectronics on a Graphene Interface Adv. Mater. 2014, 26, 482-486. 
(31) Ansari, Z. A.; Ansari, S. G.; Seo, H. K.; Kim, Y. S.; Shin, H. S. Urea Sensing Characteristics of Titanate Nanotubes Deposited by Electrophoretic Deposition Method. J. Nanosci. Nanotech 2011, 11, 3323-3329.

(32) Koncki, R.; Mohr, G. J.; Wolfbeis, O. S. Enzyme biosensor for urea based on a pH bulk optode membrane. Biosens Bioelectron. 1995, 10, 653-659.

(33) Kaushik, A.; Solanki, P. R.; Ansari, A. A.; Sumana, G.; Ahmad, S.; Malhotra, B. D. Iron oxide-chitosan nanobiocomposite for urea sensor. Sens. Act. B-Chem. 2009, 138, 572-580.

(34) Ansari, S. G.; Ansari, Z. A.; Seo, H. K.; Kim, G. S.; Kim, Y. S.; Khang, G.; Shin, H. S. Urea sensor based on tin oxide thin films prepared by modified plasma enhanced CVD. Sens. Act. B-Chem 2008, 132, 265-271.

(35) Wolfbeis, O. S.; Li, H. Fluorescence optical urea biosensor with an ammonium optrode as transducer. Biosens Bioelectron. 1993, 8, 161-166.

(36) Kovacs, B.; Nagy, G.; Dombi, R.; Toth, K. Optical biosensor for urea with improved response time. Biosens Bioelectron. 2003, 18, 111-118.

(37) Wang, J. Q.; Chou, J. C.; Sun, T. P.; Hsiung, S. K.; Hsiung, G. B. pH-based potentiometrical flow injection biosensor for urea. Sens. Act. B-Chem 2003, 91, 5-10.

(38) Sawicka, K.; Gouma, P.; Simon, S. Electrospun biocomposite nanofibers for urea biosensing. Sens. Act. B-Chem 2005, 108, 585-588. 
Scheme 1. Schematic representation of assembled CHIT-g-PPy/Urease nanostructures and optical sensing process.

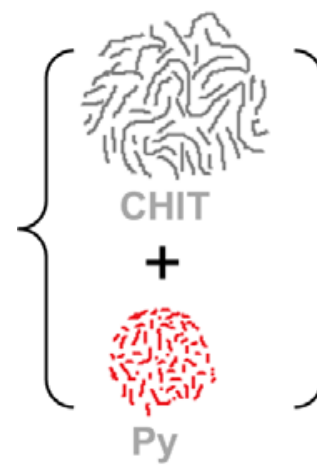

\section{Graft-} copolymerization
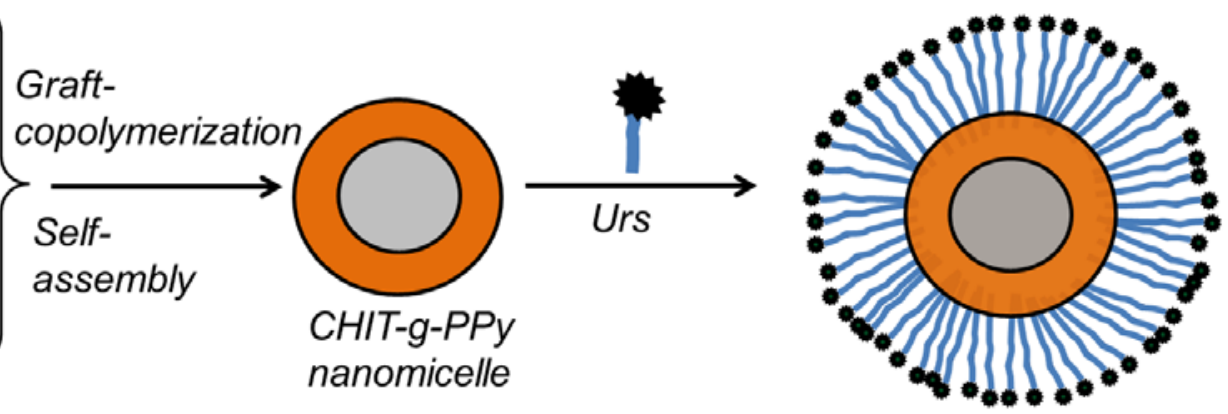

Urs/CHIT-g-PPy nanomicelle

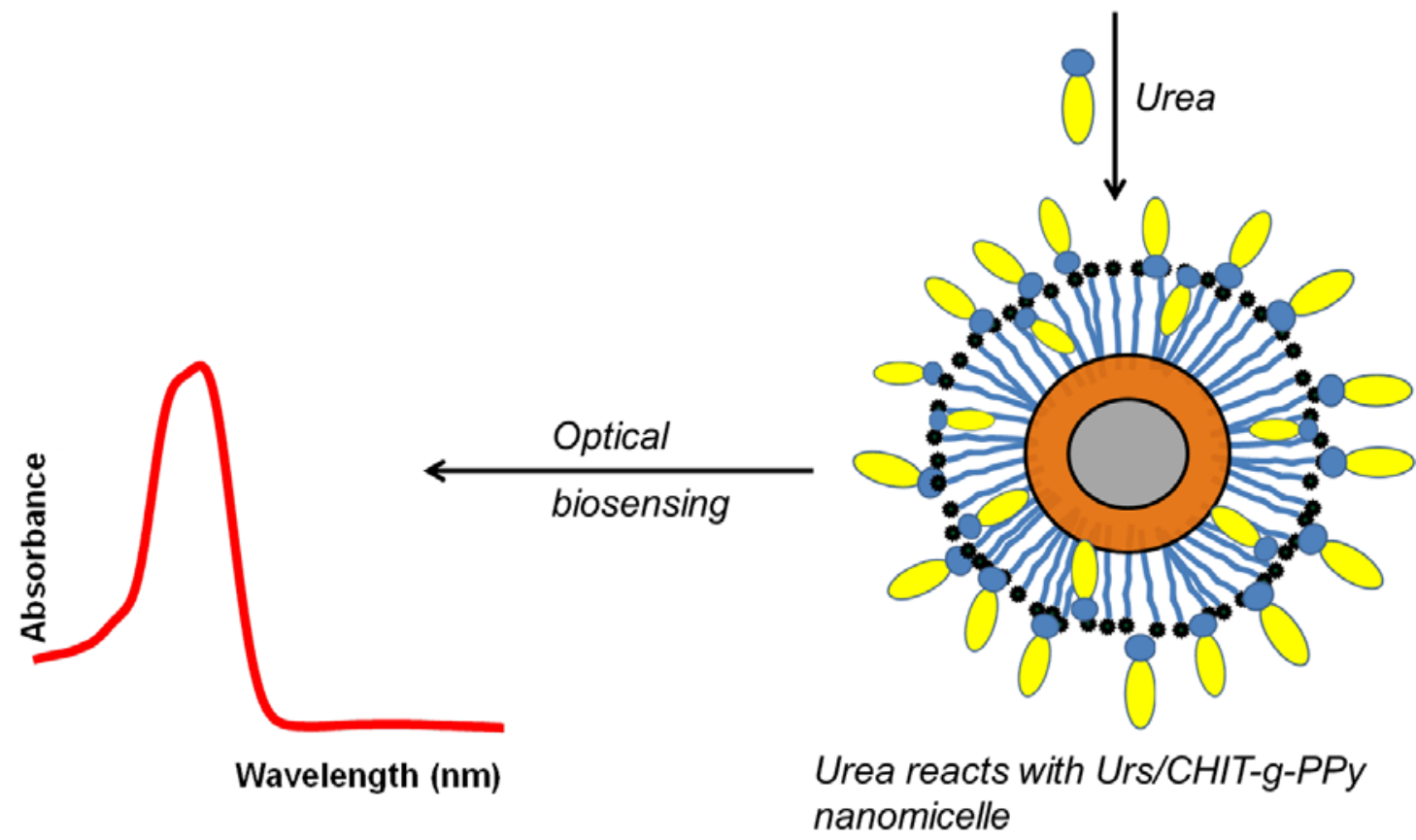


Figure 1. DLS number size distribution of micellar nanostructures before (CHIT- $g$-PPy) and after enzyme immobilisation (CHIT-g-PPy/Urs).

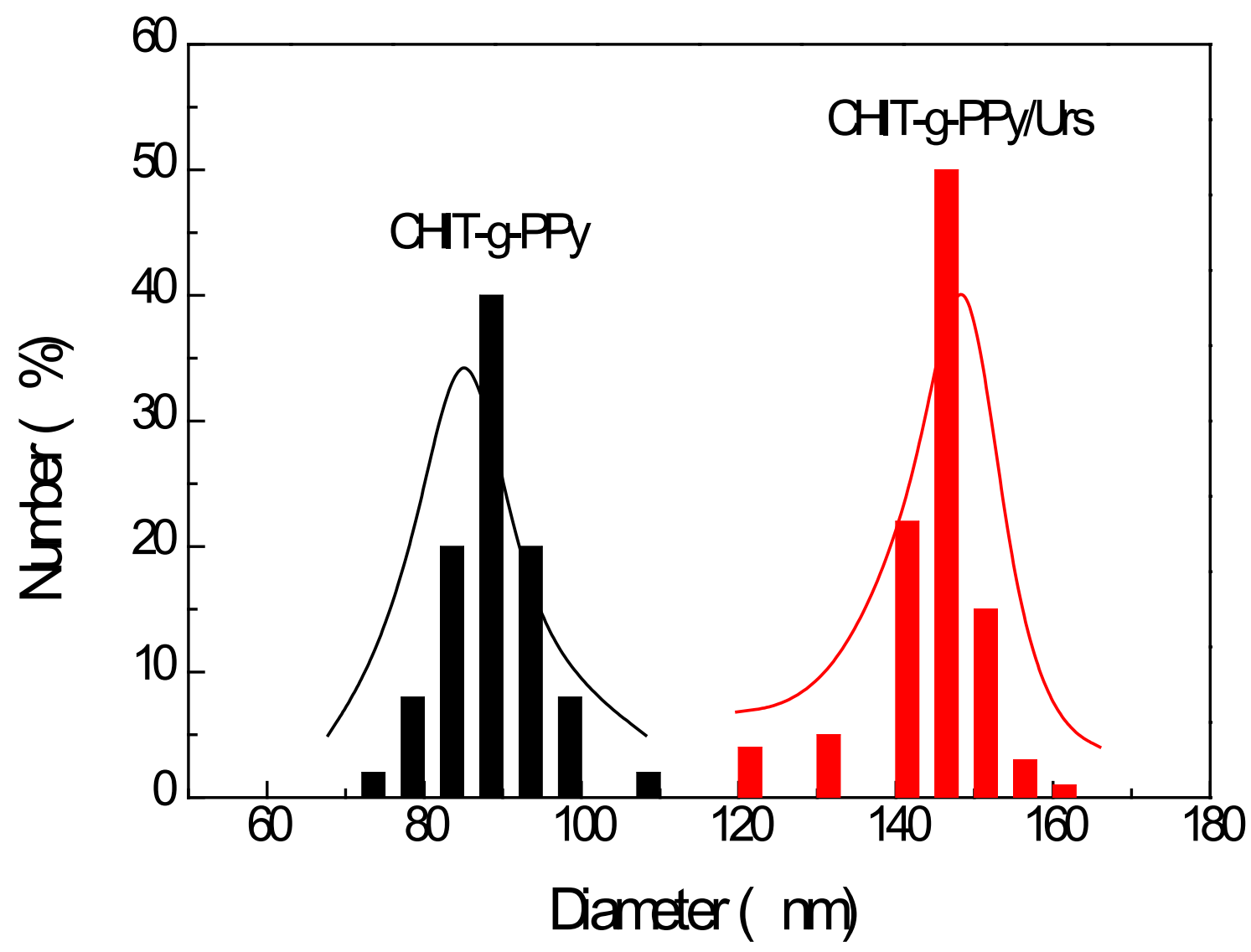


Figure 2. TEM images obtained from (a) as-synthesized CHIT-PPy and (b) enzyme-immobilized CHITPPy particles.

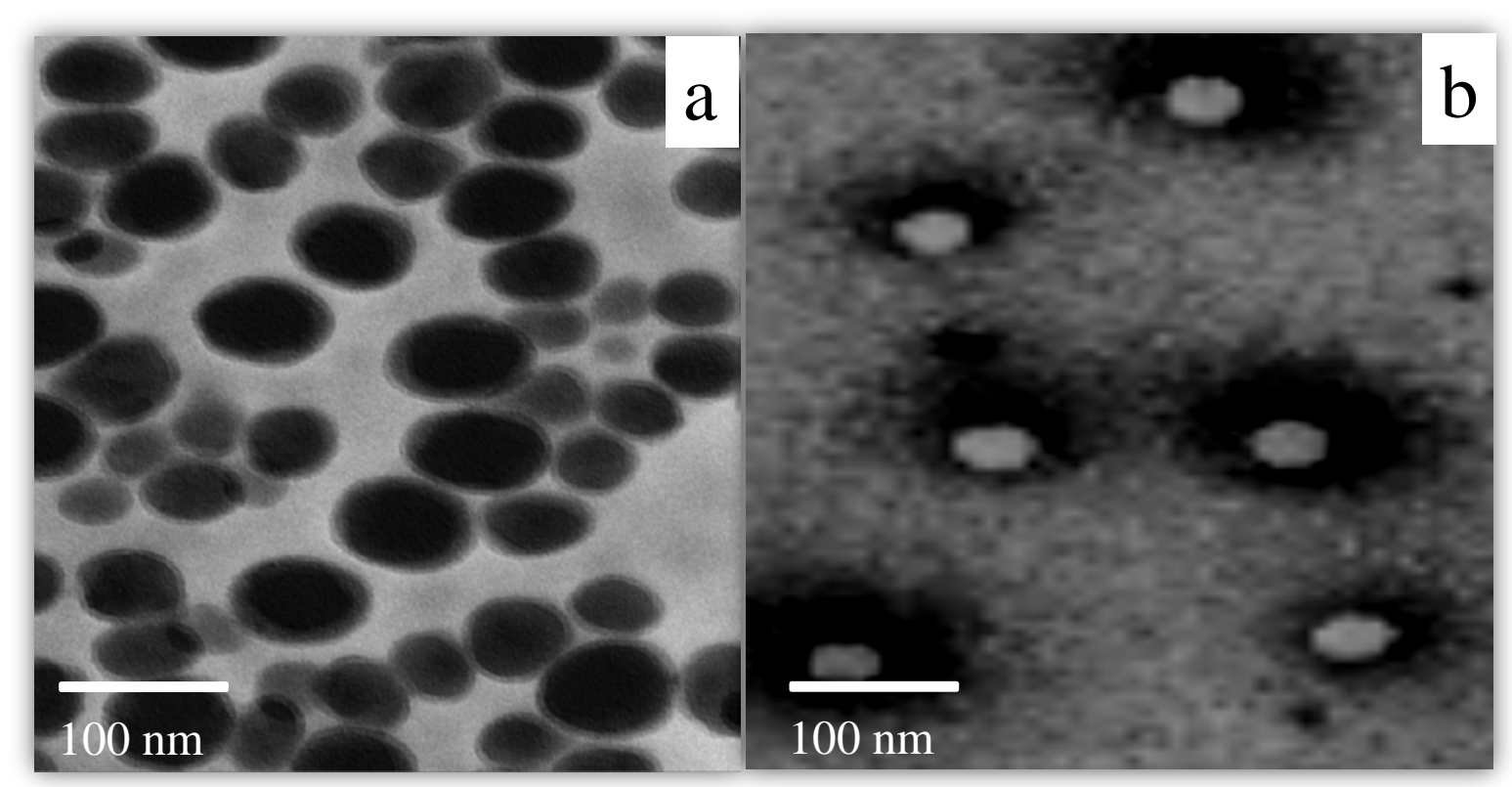


Figure 3. FTIR spectra of CHIT-PPy nanomicelles before and after enzyme immobilisation.

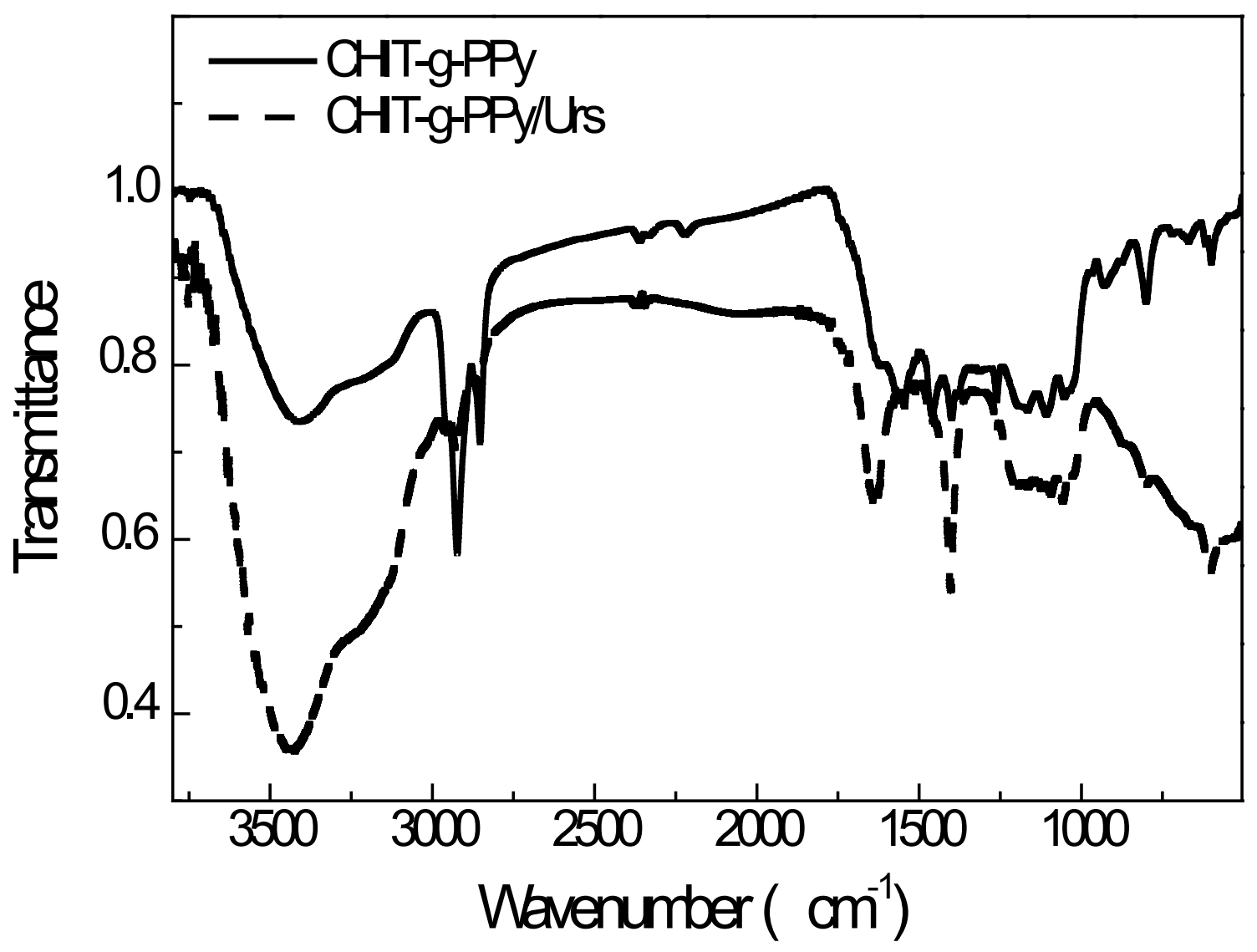


Figure 4. Photometric response of CHIT-PPy/Urs nanomicelles as a function of urea concentration (exposed with $1 \mathrm{~mL}$ of Nessler's reagent).

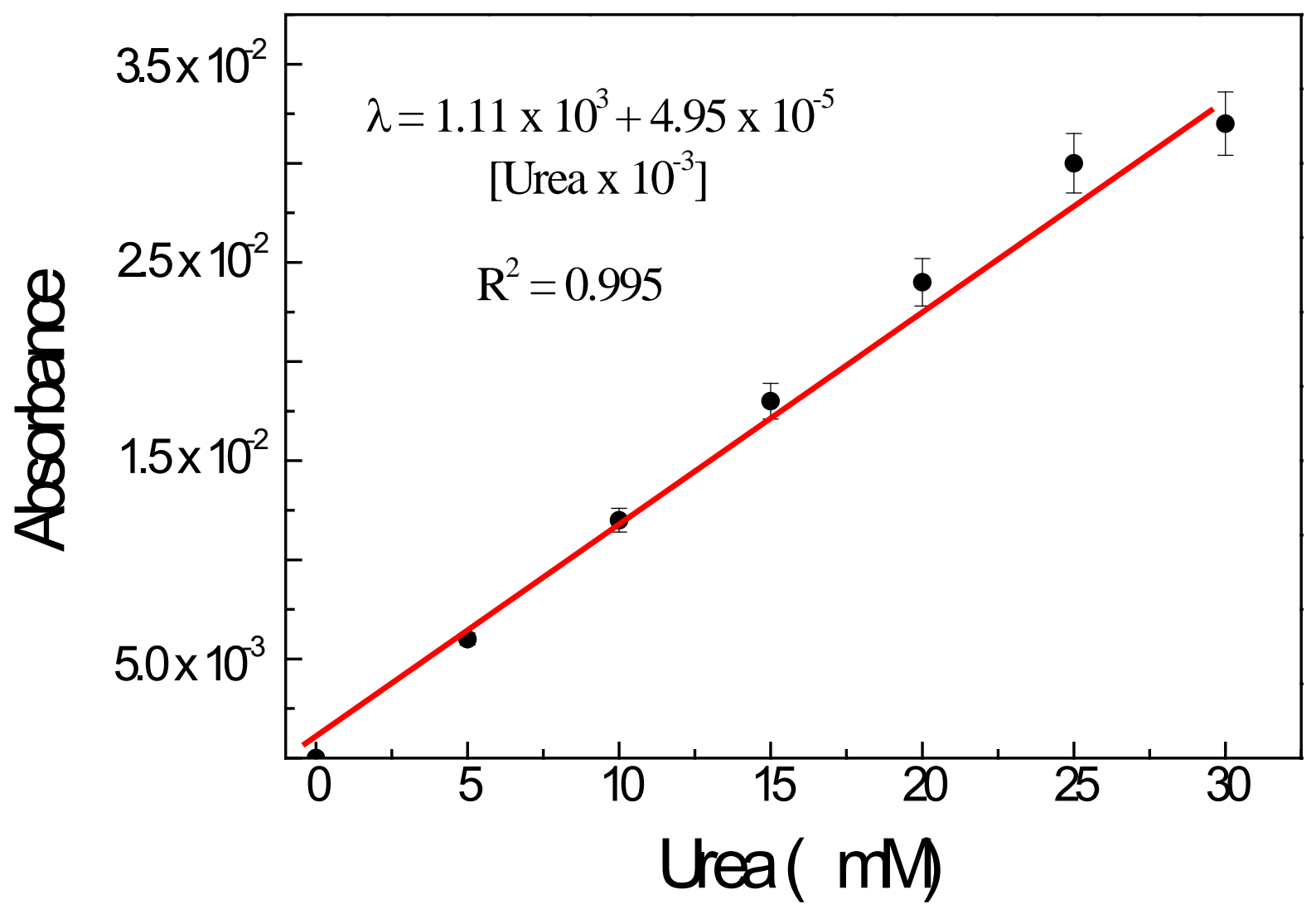


Table 1. Comparison of urea sensing properties of the current optical biosensor with previously reported values from the literature.

\begin{tabular}{|c|c|c|c|c|}
\hline Materials & Methods & Detection range & $\begin{array}{l}\text { Response } \\
\text { time }\end{array}$ & References \\
\hline CHIT-g-PPy & Optical & $0.01-30 \mathrm{mM}$ & $12 \mathrm{sec}$ & Present \\
\hline $\begin{array}{l}\text { micellar } \\
\text { nanostructure }\end{array}$ & & & & Work \\
\hline Titanate & Electrical & $1-100 \mathrm{mM}$ & - & 31 \\
\hline $\begin{array}{l}\text { Carboxy vinyl } \\
\text { chloride }\end{array}$ & Optical & 0.3 to $100 \mathrm{mM}$ & Up to $6 \mathrm{~min}$ & 32 \\
\hline$\left(\mathrm{Fe}_{3} \mathrm{O}_{4} \mathrm{NP} / \mathrm{CHIT}\right)$ & Electrical & $5-500 \mathrm{mgL}^{-1}$ & $10 \mathrm{sec}$ & 33 \\
\hline Tin oxide & Conductivity & above $50 \mathrm{mM}$ & - & 34 \\
\hline $\begin{array}{l}\text { Polyvinyl } \\
\text { chloride }\end{array}$ & Florescence & 0.03 to $0.6 \mathrm{mM}$ & $4 \mathrm{~min}$ & 35 \\
\hline Plasticized PVC & Optical & 0.1 to $1 \mathrm{mM}$ & $20 \mathrm{sec}$ & 36 \\
\hline $\begin{array}{l}\text { Photo-curable } \\
\text { gels }\end{array}$ & $\begin{array}{l}\mathrm{pH} \quad \text { based } \\
\text { Potentiometric }\end{array}$ & $30-300 \mathrm{mg} / \mathrm{dL}$ & - & 37 \\
\hline $\begin{array}{l}\text { Polyvinyl } \\
\text { pyrrolidone }\end{array}$ & Conductivity & 0.5 to $2.5 \mathrm{mM}$ & $24 \min$ & 38 \\
\hline
\end{tabular}




\section{The Table of Content}

The fabrication of a self-reporting optical urea nanobiosensor based on Urs/CHIT- $g$-PPy nanomicelles is reported. Urease is immobilised onto amphiphilic PPy grafted CHIT, and serves as a nano-matrix for the development of a urea nanobiosensor. The copolymerised CHIT-g-PPy hybrid nanomicellar structure offered a high level of enzyme immobilisation leading to highly stable Urs/CHIT-g-PPy nanomicelles to sense urea.

Keyword: Polymeric nanomicelle, graft co-polymerisation, self-reporting, urea nanobiosensor.

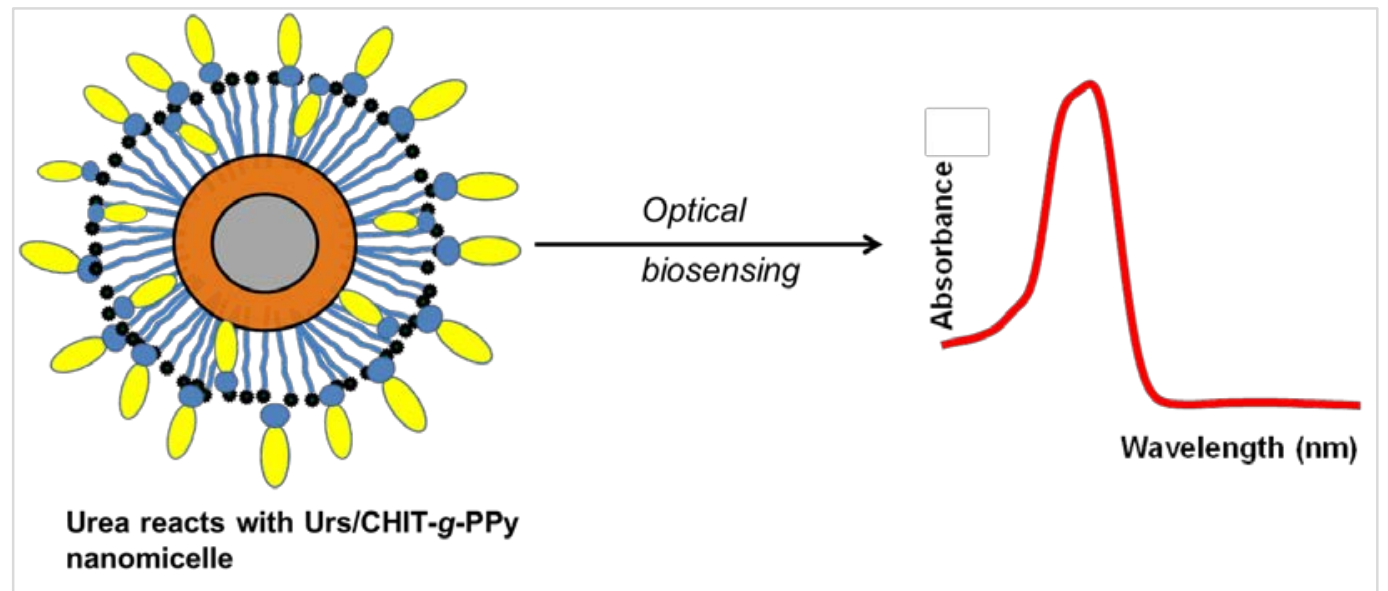

